\title{
Caracterización de la paciente obstétrica críticamente enferma, experiencia de la maternidad "Dr. Armando Castillo Plaza", Maracaibo, Venezuela: 2011 - 2014
}

\author{
María Lorena Nava ${ }^{1}$, José Ramón Urdaneta M. ${ }^{2}$, María Elena González I. 1,3, Laurenis \\ Labarca. ${ }^{1,4}$, Ángel Silva Bentacourt. ${ }^{1,4}$, Alfi Contreras Benítez ${ }^{4,5}$, Mariem Fernández. ${ }^{4}$, \\ Nasser Baabel Zambrano. ${ }^{3}$
}

${ }^{1}$ Médico Adjunto Servicio Autónomo Hospital Universitario de Maracaibo. ${ }^{2}$ Profesor Escuela de Bioanálisis. Facultad de Medicina. Universidad del Zulia. ${ }^{3}$ Profesor Escuela de Medicina. Facultad de Medicina. Universidad del Zulia. ${ }^{4}$ Alumno Doctorado en Ciencias Médicas. Facultad de Medicina. Universidad del Zulia. ${ }^{5}$ Profesor Cátedra de Anatomía. Facultad de Odontología. Universidad del Zulia. Maracaibo, Venezuela.

\section{RESUMEN}

Objetivo: Caracterizar las pacientes obstétricas críticamente enfermas asistidas en la Unidad de Cuidados Intensivos Obstétricos (UCIO) de la Maternidad "Dr. Armando Castillo Plaza" de Maracaibo, estado Zulia, durante 2011-2014. Métodos: Investigación de tipo descriptiva con diseño retrospectivo, la cual incluyó una muestra por cuotas de todas las embarazadas críticamente enfermas que ingresaron en la UCIO; se analizaron las características asociadas a la morbilidad materna extrema y la mortalidad asociada a la misma. Resultados: En el periodo estudiado, 0,74\% (548/73.588) de todos los ingresos obstétricos ingresaron a la UCIO, principalmente debido a trastornos hipertensivos $(53 \%)$, infecciones $(23,27 \%)$ o hemorragias (13,79\%). Entre los factores de riesgo encontrados destacan: edad entre $19-35$ años $(58,19 \%)$, procedencia rural (59,05\%), bajos niveles educativos ( $>80 \%$ escolaridad menor a secundaria), concubinas $(60,78 \%)$, control prenatal ausente o inadecuado $(74,57 \%)$, multiparidad $(37,93 \%)$, embarazo pretérminos $(65,95 \%)$, realización de cesárea segmentaria $(62,50 \%)$, presencia de enfermedad de la gestación $(59,05 \%)$ e ingreso durante el puerperio $(80,17 \%)$. Se encontró que la estancia hospitalaria en la UCIO fue corta $(5,91 \pm 8,17$ días), siendo las disfunciones más frecuentes la cardiovascular $(50,43 \%)$, hematológica $(41,37 \%)$ o respiratoria $(31,46 \%)$. Se registraron 38 muertes maternas entre todas las pacientes con morbilidad materna extrema ingresadas a la UCIO, con una tasa de letalidad de 6,93\%. Conclusión: Si bien las pacientes obstétricas críticamente enfermas representan menos del $1 \%$ de todos los ingresos efectuados, contribuyeron con más de la mitad de la mortalidad materna registrada en la institución.

\section{PALABRAS CLAVE: Embarazada crítica, morbilidad materna extrema, mortalidad materna}

\section{SUMMARY}

Aim: To characterize the critically ill obstetrical patients assisted in the Obstetric Intensive Care Unit (OICU) at the maternity "Dr. Armando Castillo Plaza" in Maracaibo, Zulia state, Venezuela, during 2011-2014. Methods: A descriptive research with retrospective design was developed, which included a quota sample of all critically ill pregnant women who joined the OICU; the characteristics associated with extreme maternal morbidity and mortality associated with it were analyzed. Results: In the study period, $0.74 \%(548 / 73,588)$ of all obstetric patients was admitted to the OICU, mainly due to hypertensive disorders $(53 \%)$, infections $(23.27 \%)$ or bleeding (13.79\%). Among the risk factors found are: age between $19-35$ years $(58.19 \%)$, rural 
origin (59.05\%), low educational levels (>80\% lower secondary), concubines $(60.78 \%)$, absent or inadequate prenatal care $(74.57 \%)$, multiparity $(37.93 \%)$, preterm pregnancy $(65.95 \%)$, performing segmental cesarean section (62.50\%), presence of gestation illness (59.05\%) and income during the postpartum period $(80.17 \%)$. It was found that the hospital stay in OICU was short $(5.91 \pm 8.17$ days); the most frequent dysfunctions were: cardiovascular $(50.43 \%)$, hematological $(41.37 \%)$ or respiratory $(31.46 \%)$. Were recorded 38 maternal deaths among all patients with extreme maternal morbidity admitted to OICU, with a fatality rate of $6.93 \%$. Conclusion: Although critically ill obstetric patients represent less than $1 \%$ of all obstetrics patients admitted made contributed more than half of maternal deaths recorded in the institution.

\section{KEY WORDS: Critical ill pregnant, extreme maternal morbidity, maternal mortality}

\section{INTRODUCCIÓN}

A principios del siglo pasado, un número considerable de embarazadas morían a consecuencia de la gestación, identificándose claramente la hemorragia, la infección y la preeclampsia como las causas principales de morbimortalidad materna. A cien años, en los albores de este nuevo siglo, la mortalidad materna (MM) aún puede considerarse un problema de salud pública en muchos países (1). Pese a los avances terapéuticos de esta época, mundialmente cada año siguen falleciendo alrededor de 529.000 mujeres (2-4).

Si hay un indicador que califique, sin sesgos cualquier sistema sanitario y las políticas de salud pública de un país, es a través de la determinación de las tasas de MM, sin embargo, la MM en los países emergentes no ha mostrado mayor reducción ni indicios de mejorar (5). Se ha establecido que el $99 \%$ de las muertes maternas anuales estimadas ocurren en países sub-desarrollados (6); no obstante, países en desarrollo como Chile presentan tasas de MM bajas, lo cual se ha logrado con un compromiso de políticas sólidas y modelos de atención específicos (7).

En los países del primer mundo, la MM es una complicación cada vez más rara del embarazo (5). En Venezuela se desconoce el panorama actual de esta problemática, dado el retardo en la publicación de los anuarios de mortalidad por parte de las autoridades gubernamentales; algunos investigadores han reportado que el país aporta alrededor de 300 de las 23.000 muertes maternas anuales que suceden en Latinoamérica (8) y las tasas de MM se mantienen relativamente constantes y elevadas, a pesar de que la meta trazada para el año 2015, era la reducción de la MM hasta alcanzar cifras de 14,8 por 100.000 NV (9).

La MM es sólo la punta visible del problema, puesto que por cada muerte, también hay un alto porcentaje que presentan algún tipo de comorbilidad y complicaciones graves, que requieren atención en Unidades de Cuidados Intensivos (UCI) generales o especializadas en la atención de la paciente obstétrica críticamente enferma (10). La creación de $\mathrm{UCl}$ obstétricas ha sido la forma de responder al reto implícito de estas condiciones agudas, pues al disponer de mecanismos e instrumentos que permitan tener mayor capacidad de respuesta para mejorar, no sólo la atención prenatal, sino también el entorno en que se desarrolla el evento obstétrico, y las complicaciones médicoobstétrico-quirúrgicas, la atención a la mujer embarazada mejora sustancialmente (11).

La admisión temprana de gestantes críticas en unidades preparadas para la monitorización materna y fetal continua disminuye el riesgo de progresión y severidad de la morbilidad materna extrema (6), en tanto que la carencia de $\mathrm{UCl}$ en pacientes con indicaciones claras de éstos, aumenta considerablemente la MM, puesto que un ingreso temprano es fundamental para minimizar el deterioro y la falla orgánica, determinantes importantes de la estancia posterior en UCI (12).

La evolución del cuidado crítico para pacientes obstétricas ha seguido las mismas pautas de desarrollo, y se ha convertido en una de las áreas de desarrollo en medicina materno-fetal (13). Aunque la prevalencia de pacientes obstétricas que son admitidas a UCI es difícil de precisar, se calcula que las pacientes que ameritan cuidado crítico durante el embarazo varía entre 1 a 9 pacientes por 1.000 embarazos (6). En países desarrollados se estima que la paciente embarazada representa $1-2 \%$ de las admisiones a la $\mathrm{UCl}$ y en países sub-desarrollados puede aumentar hasta $10 \%$ debido a condiciones socioculturales y económicas (10).

Recientemente, se ha determinado que la tasa de admisión de pacientes obstétricas a la $\mathrm{UCI}$ se encuentra entre $0,9 \%$ y $1,84 \%$ del total de embarazos (14). A su vez, Zwart y cols (15) plantean que la tasa de ingresos está alrededor de 0,8 por 1.000 nacidos vivos, y otras publicaciones establecen que la tasa de ingresos de pacientes obstétricas a la $\mathrm{UCl}$ se encuentra en 0,4 a 0,6 por 1.000 nacidos vivos para Estados Unidos, y 0,1-0,3 por 1.000 nacidos vivos para Canadá (16), mientras que en India se estima en 2-4 por cada 1.000 nacimientos 
(17). La admisión de gestantes críticas es un reto tanto para los médicos obstetras como intensivistas debido a que los cambios fisiológicos de múltiples órganos durante los períodos de embarazo y posparto provocan que algunas enfermedades sean más complicadas, por tanto, con el fin de reducir la mortalidad se requiere un seguimiento intensivo especial para las embarazadas en estado crítico (18).

En virtud delos planteamientos anteriormente señalados y del repunte de la MM en el país y en el estado, surgió la necesidad de investigar las características de las pacientes obstétricas críticas atendidas en la Unidad de Cuidados Intensivos Obstétricos de la Maternidad "Dr. Armando Castillo Plaza" durante los años 2011-2014, en Maracaibo, estado Zulia, Venezuela.

\section{PACIENTES Y MÉTODOS}

Investigación de tipo descriptiva y exploratoria, con diseño retrospectivo, donde se analizaron los expedientes clínicos de todas las pacientes obstétricas ingresadas a la UCIO de la Maternidad "Dr. Armando Castillo Plaza" del Servicio Autónomo Hospital Universitario de Maracaibo (SAHUM) durante el periodo 2011-2014, población que correspondió a 548 pacientes. De este universo, se determinó una muestra, con un nivel de confianza de $95 \%$ y error de $5 \%$, de 232 pacientes y mediante un muestreo aleatorio e intencionado por cuotas representativas según el número de ingresos anual, quedó distribuidas de la siguiente manera: 69/162 $(29,56 \%)$ en $2011,63 / 148(27,0 \%)$ en $2012,57 / 135$ $(24,65 \%)$ en 2013 y $103 / 43(18,79 \%)$ en 2014 . Para la conformación de la muestra, a cada paciente se le asignó un número consecutivo desde la primera paciente ingresada en el año 2011 hasta la última del año 2014 y mediante una tabla de números aleatorios se seleccionaron las pacientes hasta completar la cuota correspondiente para cada año.

Se procedió a asentar en una ficha de registro diseñada ad hoc las características generales de las pacientes: (a) edad, (b) estancia hospitalaria en UCI y sala de hospitalización y (c) estado de la paciente al ingreso (embarazada o puérpera). Seguidamente se analizaron las siguientes cualidades: A. Morbilidad: se establecieron los diagnósticos de ingreso de las pacientes a la institución, bien sea: hemorragias, infecciones, hipertensión arterial, u otras causas. B. Factores de riesgos: tales como la procedencia (rural o urbana), estado civil (soltera, casada, divorciada o viuda), escolaridad (último nivel académico alcanzado), control prenatal (adecuado si al momento del evento la paciente había asistido a por lo menos 3 consultas de atención prenatal), paridad (primiparidad o multiparidad), edad materna (precoz menor a 19 años o avanzada mayor a 35 años), edad gestacional (pretérmino menor a 37 semanas, a término entre 37 y 42 semanas y postérmino mayor a 42 semanas), tipo de embarazo (simple o gemelar), tipo de parto (eutócico o distócico), hábitos (alcohol, tabaco, drogas ilícitas), antecedentes mórbidos (enfermedad propia de la gestación o condición médica preexistente definida como cualquier enfermedad o síndrome, actual o pasado, que conste en la historia clínica o refiera la paciente o su familia al ingreso, por la que pudo haberse estudiado, recibido o no tratamiento ambulatorio o en internación, incluyendo tratamientos quirúrgicos). C. Complicaciones, agrupadas por sistemas: Disfunción cardiovascular (hipotensión debida a hipovolemia, vasodilatación periférica con descenso de los índices de resistencia e impacto en la función ventricular izquierda), Disfunción respiratoria (descenso en la $\mathrm{PaO} 2$, taquipnea y signos de edema pulmonar), Disfunción gastrointestinal (disminución en la perfusión de la mucosa intestinal, intolerancia al soporte nutricional, úlceras de estrés, pancreatitis y disfunción hepática), Disfunción renal (disminución en el flujo sanguíneo renal con oliguria y elevación de azoados), Disfunción hematológica (cambios en el recuento de glóbulos blancos por leucopenia o leucocitosis severa, anormalidades en la coagulación y coagulación intravascular diseminada), Disfunción del sistema nervioso central (alteración del estado de conciencia y coma), o falla orgánica múltiple (disfunción de 2 o más órganos o sistemas). D. Tratamiento: intervenciones realizadas, definidas como soporte inotrópico, ventilatorio, transfusiones de hemoderivados, hipotensores (sulfato de magnesio u otros antihipertensivos), sedación-analgesia, cirugía (histerectomía u otras intervenciones quirúrgicas). E. Tasa de mortalidad: definida como el número de muerte de una mujer durante el embarazo, parto o puerperio, calculadas de manera global y de acuerdo a si eran muertes maternas directas o indirectas.

Los registros obtenidos fueron procesados por medio del Paquete Estadístico para Ciencias Sociales (SPSS), versión 19, efectuándose un tratamiento estadístico de tipo descriptivo, donde los datos se expresaron mediante frecuencias absolutas o relativas (porcentajes), medidas de tendencia central (medias) y de dispersión (desviación estándar). Asimismo se determinaron las tasas de mortalidad materna, expresadas en proporción a 100.000 nacidos vivos.

\section{RESULTADOS}

Durante el periodo 2011-2014 ingresaron a la institución 73.588 pacientes por causas obstétricas, de las cuales $0,74 \%(n=548)$ ameritaron ser recibidas en UCIO. Dentro de las causas de morbilidad al ingreso a la UCIO (Tabla I), destaca la hipertensión con una frecuencia del 50\% (32/116), sin embargo al totalizar los casos acompañados con otra patología esta ascienden al 53\%. 
Seguidamente, se encontraron las infecciones $(23,27 \%)$ y las hemorragias $(13,79 \%)$, estas tres en su conjunto superan el $90 \%$ de los motivos de ingreso a la UCIO.

En cuanto a las características demográficas encontradas en la muestra analizada (Tabla II), destacaron la presencia principalmente de mujeres adultas jóvenes entre los 19 y 35 años (58,19\%), de procedencia rural $(59,05 \%)$, con bajos niveles educativos ( $>80 \%$ escolaridad menor de educación secundaria) y sin unión conyugal legal $(>80 \%$ concubinas o solteras). Respecto a los factores de riesgos clínicos presentes en las pacientes obstétricas críticas se presentan en la Tabla III, nótese que destacan la presencia de pacientes con control prenatal inadecuado $(74,57 \%)$, multíparas $(37,93 \%)$, con predominio de embarazo simples $(99,14 \%)$ y pretérminos $(65,95 \%)$; asimismo, a la mayoría de estas se les había realizado cesárea segmentaria $(62,50 \%)$, presentaron alguna enfermedad de la gestación $(59,05 \%)$ e ingresaron a la UCIO durante el puerperio $(80,17 \%)$.

Tabla I

MORBILIDAD MATERNA EXTREMA EN PACIENTES OBSTÉTRICAS CRÍTICAS UCIO - MATERNIDAD “DR. ARMANDO CASTILLO PLAZA”, 2011- 2014

\begin{tabular}{lrc}
\hline Causas & $\mathrm{n}$ & $\%$ \\
\hline Hipertensión Arterial & 116 & 50,00 \\
$\bullet$ & 55 & 23,72 \\
• Síndrome HELLP & 38 & 16,37 \\
• Hipertensión crónica & 23 & 09,91 \\
Infecciones & 54 & 23,27 \\
Hemorragia & 32 & 13,79 \\
Insuficiencia respiratoria & 8 & 3,45 \\
Neoplasias & 6 & 2,59 \\
Epilepsia & 5 & 2,16 \\
Embolismo pulmonar & 3 & 1,29 \\
Obesidad mórbida & 1 & 0,43 \\
Hipertensión + Hemorragia & 5 & 2,16 \\
Hipertensión + Infección & 2 & 0,86 \\
\hline
\end{tabular}

$n=232$
Con relación a la estancia hospitalaria, se evidenció que la estancia en la UCIO fue entre 1 y

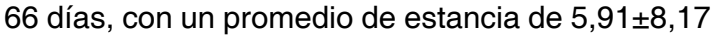
días; mientras que la estancia en sala de hospitalización en la maternidad fue en promedio de

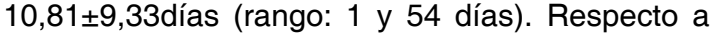
las complicaciones presentadas (Tabla IV), destacaron principalmente las disfunciones cardiovascular $(50,43 \%)$, hematológica $(41,37 \%)$ y respiratoria $(31,46 \%)$.

Por último, en cuanto a la mortalidad materna, en la Tabla $\mathrm{V}$ se observa una tasa de letalidad de casi $7 \%$ entre las pacientes con morbilidad materna extrema ingresadas a la UCIO y que fallecieron por causas relacionadas con el embarazo, parto o puerperio, observándose con preocupación una tendencia en su incremento. Por otra parte, al compararse con la mortalidad materna de toda la Maternidad "Dr. Armando Castillo Plaza", las muertes presentadas en la UCIO representaron casi las $2 / 3$ partes $(65,52 \%)$, evidenciándose tasas de mortalidad materna constantemente elevadas durante el periodo estudiado.

\section{Tabla II \\ FACTORES DE RIESGO DEMOGRÁFICOS EN PACIENTES OBSTÉTRICAS CRITICAS. UCIO - MATERNIDAD "DR. ARMANDO CASTILLO PLAZA", 2011 - 2014}

\begin{tabular}{lrr}
\hline Factor de riesgo & $\mathrm{n}$ & $\%$ \\
\hline Edad Materna ( años) & & \\
- Precoz ( $\leq 18)$ & 65 & 28,01 \\
- Adecuada (19-35) & 135 & 58,19 \\
- Avanzada ( $\geq 36)$ & 32 & 13,80 \\
Procedencia & & \\
- Rural & 137 & 59,05 \\
- Urbana & 95 & 40,95 \\
Estado Civil: & & \\
- Soltera & 49 & 21,12 \\
- Casada & 42 & 18,10 \\
- Concubinato & 141 & 60,78 \\
Escolaridad: & & \\
- Analfabeta & 33 & 14,22 \\
• Primaria completa & 70 & 30,17 \\
- Secundaria completa & 83 & 35,78 \\
• Técnico & 30 & 12,93 \\
- Universitario & 16 & 6,90 \\
\hline $\mathrm{n}=232$ & & \\
\hline
\end{tabular}




\section{Tabla III \\ FACTORES DE RIESGOS CLÍNICOS EN PA- CIENTES OBSTÉTRICAS CRÍTICAS. \\ UCIO - MATERNIDAD “DR. ARMANDO CASTILLO PLAZA", 2011- 2014}

\begin{tabular}{|c|c|c|}
\hline Factor de riesgo & $\mathrm{n}$ & $\%$ \\
\hline \multicolumn{3}{|l|}{ Control prenatal: } \\
\hline - Inadecuado & 173 & 74,57 \\
\hline - Adecuado & 59 & 25,43 \\
\hline \multicolumn{3}{|l|}{ Paridad: } \\
\hline - Primiparidad & 74 & 31,90 \\
\hline - 2-4 paras & 70 & 30,17 \\
\hline - Multiparidad $(\geq 5)$ & 88 & 37,93 \\
\hline \multicolumn{3}{|l|}{ Edad gestacional: } \\
\hline - Pre-término & 153 & 65,95 \\
\hline - A término & 79 & 34,05 \\
\hline \multicolumn{3}{|l|}{ Tipo de embarazo: } \\
\hline - Simple & 230 & 99,14 \\
\hline - Gemelar & 2 & 0,86 \\
\hline \multicolumn{3}{|l|}{ Condición al ingreso: } \\
\hline - Embarazada & 46 & 19,83 \\
\hline - Puérpera & 186 & 80,17 \\
\hline \multicolumn{3}{|l|}{ Atención obstétrica : } \\
\hline - Cesárea segmentaria & 145 & 62,50 \\
\hline - Parto vaginal & 68 & 29,32 \\
\hline - Legrado uterino & 4 & 1,72 \\
\hline - Histerectomía obstétrica & 14 & 6,03 \\
\hline - Laparotomía (embarazo ectópico) & 1 & 0,43 \\
\hline \multicolumn{3}{|l|}{ Antecedentes mórbidos: } \\
\hline - Sin antecedentes & 35 & 15,09 \\
\hline - Condición preexistente & 60 & 25,86 \\
\hline - Enfermedad de la gestación & 137 & 59,05 \\
\hline
\end{tabular}

\section{DISCUSIÓN}

La paciente obstétrica críticamente enferma representa un grupo de características únicas de difícil manejo, debido a las alteraciones de la fisiología materna, las enfermedades específicas del embarazo y en muchas ocasiones la presencia fetal. El presente estudio informa que el $0,74 \%$ de las admisiones obstétricas durante 2011-2014 requirieron cuidados intensivos; dicho resultado se encuentra dentro del rango de $0,1 \%-0,8 \%$ sugerido de pacientes obstétricas admitidas a las UCI (19).

Al analizar las causas o los motivos que

\section{Tabla IV \\ COMPLICACIONES EN PACIENTES OBSTÉTRICAS CRÍTICAS. \\ UCIO - MATERNIDAD “DR. ARMANDO \\ CASTILLO PLAZA", 2011- 2014}

\begin{tabular}{lcc}
\hline Complicaciones & $\mathrm{n}$ & $\%^{*}$ \\
\hline Disfunción cardiovascular & 117 & 50,43 \\
Disfunción hematológica & 96 & 41,37 \\
Disfunción respiratoria & 73 & 31,46 \\
Disfunción renal & 66 & 28,44 \\
Disfunción neurológica & 21 & 9,05 \\
Disfunción gastrointestinal & 07 & 3,01 \\
Fallo orgánico múltiple & 09 & 3,87 \\
\hline
\end{tabular}

* El porcentaje se estima de acuerdo al total de pacientes

conllevaron el ingreso de estas pacientes a una UCIO se evidenció que prevaleció principalmente los trastornos hipertensivos. Al respecto, una investigación realizada en esta misma institución señaló que las enfermedades hipertensivas del embarazo resultaron ser la principal causa de mortalidad materna con un $43,10 \%$ (20). Asimismo, otros estudios realizados en el país, en las ciudades de Caracas (21) y Mérida (22) también reportan que los trastornos hipertensivos del embarazo son responsable del $26,62 \%$ y $39,6 \%$ de las muertes maternas registradas, respectivamente.

De igual manera, investigadores latinoaméricanos en países como Colombia $(10,23,24,25)$, México $(26,27)$ o Chile $(28,29)$, han determinado que los desordenes hipertensivos representan la primera causa de mortalidad materna. Si se compara con investigaciones pasadas realizadas en Venezuela, se evidencia que los trastornos hipertensivos del embarazo han venido a repuntar como causal de muerte materna; pues en el pasado se señalaron a las hemorragias y a la sepsis como la principal causa de muerte materna en Maracaibo (30) y Caracas (31), respectivamente.

Contrariamente, otras investigaciones han establecido que la primera causa de ingreso a $\mathrm{UCI}$ dentro de las admisiones obstétricas era la hemorragia posparto; reportandose un 44,5\% en Argentina (32) y $23 \%$ en Perú (19). Asimismo, estudios realizados en Cuba determinaron que la morbilidad obstétrica extremadamente grave más frecuente era la hemorragia masiva relacionada predominantemente con la atonía uterina $(12,33)$; mientras que otros autores (34) señalan que la incidencia de ingresos de pacientes obstétricas críticas en la $\mathrm{UCl}$ se comporta de forma irregular, siendo las causas no obstétricas las que con mayor frecuencia motivaron su admisión en esta unidad. 


\section{Tabla V}

MORTALIDAD MATERNA EN PACIENTES OBSTÉTRICAS CRÍTICAS.

UCIO - MATERNIDAD “DR. ARMANDO CASTILLO PLAZA”, 2011- 2014

\begin{tabular}{|c|c|c|c|c|c|c|}
\hline \multirow[t]{2}{*}{ Año } & \multicolumn{3}{|c|}{ Mortalidad Materna Global } & \multicolumn{3}{|c|}{ Mortalidad Materna en UCIO } \\
\hline & $\mathrm{MM}^{*}$ & $N V^{\alpha}$ & $\mathrm{TMM}^{\Phi}$ & Ingresos & $\mathrm{MMU}^{\ddagger}$ & $\%^{\neq}$ \\
\hline 2011 & 4 & 12.018 & 33,28 & 162 & 1 & 1,62 \\
\hline 2012 & 18 & 16.117 & 111,68 & 148 & 11 & 7,43 \\
\hline 2013 & 20 & 16.135 & 123,95 & 135 & 13 & 9,63 \\
\hline 2014 & 16 & 14.909 & 107,31 & 103 & 13 & 12,62 \\
\hline Total & 58 & 59.179 & 98,00 & 548 & 38 & 6,93 \\
\hline
\end{tabular}

${ }^{\star} \mathrm{MM}=$ Muertes maternas en toda la institución

a $N V=$ Nacidos vivos

ФTMM $=$ Tasa de mortalidad materna por 100.000 nacidos vivos

$\ddagger \mathrm{MMU}=$ Muertes maternas en UCIO

$\neq$ Tasa de letalidad= Número de muertes en base al total de ingresos a UCIO en cada año

En cuanto a los factores de riesgos de tipo demográficos identificados se encontró una prevalencia de adolescentes embarazadas, de procedencia rural, concubinas y analfabetas o con sólo estudios primarios aprobados (44,39\%). Respecto a la edad, se sabe que las edades extremas (adolescentes y mayores de 35 años) estan asociadas a un mayor riesgo de morbi-mortalidad materna; asimismo, suele ocurrir un mayor número de nacimientos en mujeres menores de 20 y mayores de 34 años, lo cual aumenta el riesgo de complicaciones y muerte en estos grupos (2).

La Maternidad "Dr. Armando Castillo Plaza" es el principal centro de referencia del estado Zulia y la única institución de atención materna en la región que cuenta con una UCIO, motivo por los cuales recibe pacientes de diversas partes de la ciudad, del estado e inclusive de otras regiones del país. Esta diversidad de procedencias influye en la mayor prevalencia tanto de morbilidad materna extrema como de mortalidad materna, puesto que las poblaciones foráneas se ven afectadas por la accesibilidad de la atención médica en los centros de salud que implica el lugar de procedencia y la distancia con los hospitales pilotos, aunado al difícil acceso que tienen estas mujeres a la actualización tecnológica y condiciones personales y calidad de vida en su entorno social (20).

Con relación a los factores de riesgo clínicos, se encontró que la mayoría de las pacientes recibidas en la UCIO presentaba una enfermedad de la gestación $(59,05 \%)$, principalmente preeclampsia, o tenían una condición médica preexistente (25,86\%). Este hallazgo, es coincidente con el encontrado por una investigadora argentina (35) quien señaló que el $20 \%$ de las gestantes críticas había presentado alguna condición médica preexistente, mientras que la enfermedad hipertensiva en embarazo previo estuvo presente en el $20 \%$.

Otro factor frecuentemente encontrado fue el control prenatal ausente o inadecuado $(74,57 \%)$. Al respecto, investigadores como Rojas y cols (24) también señalan la ausencia de atención y cuidados médicos antenatales en casi la mitad de las gestantes críticas (49,5\%); en tanto que Gálvez y cols (10) encontraron que sólo $22 \%$ de las pacientes ingresadas en la UCI de un hospital de la capital colombiana había recibido un adecuado control prenatal.

De igual manera, esta investigación refleja que la mayoría de las gestantes en estado crítico se encontraba en el puerperio al momento de su ingreso a la $\mathrm{UCl}$, Coincidentemente, se ha señalado que la mayor parte de las gestantes críticas ingresaban a la $\mathrm{UCl}$ en el puerperio, siendo la mortalidad mayor en el período post-parto (36); asimismo, otros autores (24) encontraron que sólo el $14 \%$ de las pacientes obstétricas críticas se encontraban embarazadas al ingresar a la $\mathrm{UCl}$.

Los resultados presentados también destacan que en la mayoría de los casos, el embarazo se había interrumpido antes del término y a través de la operación cesárea. Este hallazgo coincide con el encontrado en otra investigación (35) donde se registra que el promedio de la edad gestacional en las embarazadas ingresadas en $\mathrm{UCl}$ era de 33 semanas, mientras que Suárez y cols (12) señalan que el parto distócico por cesárea es un evento obstétrico relacionado directamente con la morbilidad grave y la realización de histerectomías obstétricas. 
En cuanto a la estancia en $\mathrm{UCl}$ de las pacientes obstétricas, varían de acuerdo con la patología establecida y con la evolución de cada paciente; sin embargo, se sabe que tienen una estancia corta en estas unidades, en la mayoría de los casos, con un adecuado manejo, la estancia es menor que en la población general (37). En esta investigación se encontró que la estancia en UCIO fue corta, en promedio de 5,81 días, pero mucho mayor a la señalada en otros estudios que encontraron una estancia en $\mathrm{UCl}$ de 4 dias en promedio $(19,24,35)$.

Respecto a las complicaciones encontradas en estas pacientes se observó con mayor frecuencia la disfunción cardiovascular, seguida de la hematológica y respiratoria, lo cual coincide con lo encontrado por Malvino (32) quien al aplicar los criterios SOFA encontró que las principales disfunciones fueron la cardiovascular $(30,4 \%)$ y la hematológica $(26,6 \%)$. A diferencia de lo encontrado en esta serie, Olaya y cols (38), encontraron que la complicación más frecuente en pacientes obstétricas críticas debido a sepsis severa y choque séptico era la disfunción pulmonar (53,3\%).

Asimismo, también difiere de lo reportado por Soli (35) en pacientes críticas ingresadas con diagnóstico de preeclampsia-eclampsia donde la alteración de la función renal era la complicación más frecuente. Al respecto, Miranda y cols (23) señalan que en las gestantes con morbilidad materna extrema el desarrollo de disfunción de cualquier órgano y/o sistema, o la presencia de disfunción orgánica múltiple aumenta dramáticamente la posibilidad de fallecer, por tanto, su identificación temprana puede permitir derivar oportunamente a la paciente a un nivel de complejidad superior y de esta manera poder disminuir la mortalidad materna (39).

Por último, los resultados encontrados en esta investigación señalan una tasa de letalidad del $6,93 \%$ entre todas las gestantes que ingresaron a la UCIO, observándose una tendencia al aumento de las frecuencias a lo largo de los años estudiados y al comparar con las muertes maternas registradas en toda las áreas de esta institución se encontró que la mayoría de estas muertes sucedieron en la UCIO; estando más elevada que el 1,7\% encontrado en México (40) y que el 4,3\% señalado en Colombia (10). Sin embargo, resulta un poco menor al $9,6 \%$ reportado en una investigación peruana (19) y al $8,4 \%$ encontrado en Colombia (24); aunque otro estudio registró una mortalidad mucho mayor entre las embarazadas críticamente enfermas (30,3\%), la cual se asociaba significativamente con un bajo puntaje en la escala de Glasgow y a alta puntuación en la escala de APACHE II al ingreso (36).

Como demuestran los resultados presentados, esta investigación pone en evidencia el gravísimo problema de la morbilidad materna extrema; problemática que tal como señalan otros autores impera la necesidad de aumentar el funcionamiento de unidades de cuidados intensivos obstétricos, definir criterios de ingreso, difundir la importancia del control prenatal y la prevención y diagnóstico oportuno de los trastornos hipertensivos del embarazo (41-47). No obstante, dado el carácter retrospectivo del estudio, se tuvo como limitante que los datos asentados en las historias clínicas en ocasiones se encontraban incompletos debido a que las mismas fueron llenadas erroneamente, asimismo, algunas historias se encontraban extraviadas pues habían sido archivadas incorrectamente.

\section{CONCLUSIÓN}

Las pacientes obstétricas críticamente enfermas representaron menos del $1 \%$ de todos los ingresos efectuados y se caracterizaron principalmente por ser mujeres provenientes del medio rural, concubinas, con bajos niveles educativos, multíparas, que ingresaron por trastornos hipertensivos, con embarazos no controlados y que presentaron enfermedades de la gestación, y que contribuyeron con más de la mitad de la mortalidad materna registrada en la institución durante el periodo analizado. Resulta imprescindible que cada centro de atención materna disponga de un equipo multidisciplinario de especialistas en cuidado crítico obstétricos y en medicina materno-fetal para el difícil entendimiento y manejo de la historia natural de las enfermedades y sus complicaciones durante la gestación, el parto y el puerperio, de manera de poder tomar decisiones acertadas y oportunas para el manejo de las pacientes obstétricas críticas que permitan la reducción de la mortalidad materna.

\section{REFERENCIAS}

1. Díaz de León MA, Briones JC. Medicina Crítica en Obstetricia, una nueva especialidad. Cir Ciruj. 2006;74(4):223-4.

2. Ocanto L. La Mortalidad Materna en Venezuela. Fundación Escuela de Gerencia Social: Caracas, Venezuela. 2006

3. Cabezas E. Evolución de la mortalidad materna en Cuba. Rev Cub Salud Pública. 2006;32(1):1-9.

4. Faneite P. Mortalidad materna en la región bolivariana de Latino-América: área crítica. Rev Obstet Ginecol Venez. 2008;68(1):18-24.

5. Lozano A, Olaya SX. La importancia en Colombia de la Medicina Crítica en Obstetricia; más allá de las palabras. Acta Colomb Cuid Intensivo. 2012;12(4):199201.

6. Escobar MF. Cuidado crítico obstétrico: ¿cuál es el concepto? Acta Colomb Cuid Intensivo. 2013;13(3):180-8.

7. Pazmiño S, Guzmán N. Control prenatal y su impacto en la mortalidad materna: Análisis de una tendencia, 1994-2004. Cali, Colombia. Rev Colomb Obstet Ginecol. 2009;60(1):12-8.

8. Faneite $\mathrm{P}$. Mortalidad materna y perinatal. Tendencias 1969-2004. Rev Obstet Ginecol Venez. 2006;66(2):75-9. 
9. Mazza MM, Vallejo CM, González M. Mortalidad materna: análisis de las tres demoras. Rev Obstet Ginecol Venez. 2012;72(4):233-48.

10. Gálvez ML, Arreaza M, Rodríguez JA. Mortalidad materna de pacientes atendidas en la $\mathrm{UCl}$ del Hospital Simón Bolívar, Bogotá (Colombia) 2004 - 2006. Rev Colomb Obstet Ginecol. 2009;60(2):152-8.

11. Briones JC, Díaz M, Meneses JC. Estrategias para reducir la mortalidad materna hospitalaria en el Estado de México. Rev Asoc Mex Med Crit y Ter Int. 2009;23(1):16-24.

12. Suárez JA, Gutiérrez M, Corrales A, Benavides ME, Tempo DC. Algunos factores asociados a la morbilidad obstétrica extremadamente grave. Rev Cub Obstet Ginecol. 2010;36(2):4-12.

13. Díaz de León MA, Briones JC. Medicina crítica en obstetricia (una verdad no reconocida). Rev Asoc Mex Med Crit y Ter Int. 2012;26(1):6-10.

14. Togal T, Yucel N, Gedik E, Gulhas N, Toprak HI, Ersoy MO. Obstetrics admissions to the intensive care unit in a tertiary referral hospital. J Crit Care. 2010;25:62833.

15. Zwart JJ, Dupuis JRO, Richters A, Öry F, van Roosmalen J. Obstetric intensive care unit admission: a 2 -year nationwide population based cohort study. Intensive Care Med. 2010;36(2):256-63.

16. Zhang $\mathrm{WH}$, Alexander S, Bouvier-Colle $\mathrm{MH}$, Macfarlane A, the MOMS-B GROUP. Incidence of severe preeclampsia, postpartum haemorrhage and sepsis as a surrogate marker for severe maternal morbidity in a European population-based study: the MOMS-B survey. BJOG. 2005;112(1):89-96.

17. Tempe A, Wadhwa L, Gupta S, Bansal S, Satyanarayana $\mathrm{L}$. Prediction of mortality and morbidity by simplified acute physiology score ii in obstetric intensive care unit admissions. Indian J Med Sci.2007;61(4) [Periódico en línea]. Disponible en: http://www.indianjmedsci.org. Fecha de consulta: 07-10-2015.

18. Wang YQ, Ge QG, Wang J, Niu JH, Huang C, Zhao YY. The WHO near miss criteria are appropriate for admission of ill pregnant women to intensive care units in China. Chin Med J. 2013;126(5):895-8.

19. Acho SC, Pichilingue JS, Díaz JA, García M. Morbilidad materna extrema: admisiones gineco-obstétricas en las unidades de cuidados intensivos de un hospital general. Rev Per Ginecol Obstet. 2011;57:87-92.

20. Ávila A, Gómez M, Fuentes B, Villarroel F, Urdaneta $\mathrm{J}$, Benítez $\mathrm{B}$, et al. Aspectos clínicos y socioeconómicos relacionados a la mortalidad maternal de pacientes que acudieron a la maternidad Dr. Armando Castillo Plaza en el periodo 2011-2013. Invest Clin. 2015;56(Suppl. 1):1288-92.

21. Cabrera C, Martínez MA, Zambrano G. Mortalidad materna en la Maternidad "Concepción Palacios": 2009 2013. Rev Obstet Ginecol Venez. 2014;74(3):154-61.

22. Chacón G, Monsalve N. Mortalidad materna en el Instituto Autónomo Hospital Universitario de Los Andes (IAHULA) 1974-2005. Rev Obstet Ginecol Venez. 2007;67(2):99-105.

23. Miranda J, Rojas JA, Mendivil C, Paternina Á. Disfunción orgánica en la paciente con morbilidad materna extrema: un estudio multicéntrico. Tesis de Especialización en Ginecología y Obstetricia. Facultad de Medicina. Universidad de Cartagena. 2012. [Tesis en línea]. Disponible en: http://190.27.248.91:8082/ jspui/bitstream/11227/301/1/informe\%20tesis\%20disfuncion $\% 20$ organica $\% 20$ en $\% 20$ paciente $\% 20$ con $\% 20$ mme\%20-\%20jezi.pdf. Fecha de consulta: 28-122015.

24. Rojas JA, Miranda JE, Ramos E, Fernández JC. Cuidado crítico en la paciente obstétrica. Complicaciones, intervenciones y desenlace materno-fetal. Clin Invest Gin Obst. 2011;38(2):44-9.

25. Cáceres FM, Díaz LA. La mortalidad materna en el Hospital Universitario Ramón González Valencia entre 1994-2004. Rev Colomb Obstet Ginecol. 2007;58(2):99-107.

26. Castelazo E, Monzalbo DE, López MJ, Castelazo S. Preeclampsia atípica y éxito perinatal: reporte de caso". Ginecol ObstetMex. 2014;82(1):70-4.

27. Romero G, Hernández BO. Persistencia de hipertensión en mujeres con preeclampsia. Ginecol Obstet Mex. 2011;79(10):601-6.

28. Parra M, San Martín A, Valdés E, Hasbún J, Quiroz L, Schepeler M, et al. Espectro clínico de la preeclampsia: estudio comparativo de sus diversos grados de severidad. Rev Chil Obstet Ginecol. 2007;72(3):16975.

29. Donoso E. Mortalidad Materna en Chile, 2000-2004. Rev Chil Obstet Ginecol. 2006;71(4):246-51.

30. García JE, Molina R, Cepeda M. Tasas de mortalidad materna en los hospitales de Maracaibo: 1993-2000. Rev Obstet Ginecol Venez. 2002;62(2):103-8.

31. Brito JG, Cabrera C, Gutiérrez O, Gutiérrez M, Porras F. Mortalidad materna en la Maternidad "Concepción Palacios": 1982-1991. Rev Obstet Ginecol Venez. 2007;67(1):31-9.

32. Malvino E. Morbilidad materna aguda severa y condiciones de gravedad de enfermas obstétricas al ingreso en una Unidad de Cuidados Intensivos. En: Medicina Intensiva. 2014;31(41) [Periódico en línea]. Disponible en: http://www.obstetriciacritica.com.ar/ doc/33.pdf. Fecha de consulta: 14-11-2015.

33. Dávila HL; Rodríguez M, Peña ML, García A, Rueda $R$, Matos Z. Morbilidad de la paciente obstétrica extremadamente grave en la Isla de la Juventud, 20022010. Progr Obstet Ginecol. 2013;56(6):310-5.

34. Montoya FR, León I, Hernández W, Segura A, Rodríguez G. Atienza AM. Caracterización de pacientes obstétricas críticas. Rev Cubana Med Militar. 2011;40(2):126-36.

35. Soli SB. Presentación y evolución de las pacientes ingresadas en la Unidad de Cuidados Intensivos con diagnóstico de Preeclampsia-Eclampsia. Tesis de Especialización en Clínica Médica. Facultad de Ciencias Médicas. Universidad Nacional de Rosario. 2013. [Tesis en línea]. Disponible en: http://www.clinica-unr. com.ar/Posgrado/Graduados/silvana_soli.pdf. Fecha de consulta: 19-11-2015.

36. Bhadade R, de' Souza R, More A, Harde M. Maternal outcomes in critically ill obstetrics patients: $A$ unique challenge. J Crit Care Med. 2012;16(1):8-16.

37. García M, Ontiveros M, Whizar V. Admisiones obstétricas en la unidad de cuidados intensivos de un hospital comunitario. Anest México. 2009;21(1):7-11.

38. Olaya SX, Franco DM, Cristancho M. Sepsis severa y choque séptico en pacientes obstétricas en la unidad de cuidado intensivo: características clínicas y tratamiento. Acta Colomb Cuid Intensivo. 2013;13(3):13844. 
39. Oliveira A, Parpinelli MA, Cecatti JG, Souza JP, Sousa MH. Sequential organ failure assessment score for evaluating organ failure and outcome of severe maternal morbidity in obstetric intensive care. The Scientific World Journal. 2012: 172145. [Periódico en línea]. Disponible en: http://www.ncbi.nlm.nih.gov/ pmc/articles/PMC3289867/pdf/TSWJ2012-172145. pdf. Fecha de consulta: 9-12-2015.

40. Guerrero A, Briones CG, Díaz de León M, Briones JC. Cuidados intensivos en ginecología y obstetricia en el Hospital General de México. Rev Asoc Mex Med Crit y Ter Int. 2011;25(4):211-7.

41. García V, González M, Ardila HR. Experiencia en el manejo de la hemorragia obstétrica en una unidad de alta dependencia. Acta Colomb Cuid Intensivo. 2012;12(4):256-63.

42. Basket TF. Epidemiology of Obstetric Critical Care. Best Prac Res Cl Ob.2008;22(5):763-74.
43. Sánchez HR, Pérez G, Pérez $P$, Vázquez F. Impacto del control prenatal en la morbilidad y mortalidad neonatal. Rev Med Inst Mex Seguro Soc. 2005;43(5):37780.

44. Wheatly S. Maternal critical care: what's in a name?J Obstet Anesth2010;19:353-5.

45. Rojas J, González MV, Monsalve G, Escobar MF, Vasco M. Consenso colombiano para la definición de los criterios de ingreso a unidades de cuidados intensivos en la paciente embarazada críticamente enferma. Rev Colomb Obstet Ginecol. 2014; 65(1):47-74.

46. Trikha A, Singh PM. The critically ill obstetric patient - Recent concepts. Indian J Anaesth. 2010;54(5):4217.

47. Navas F, Pareja M, Meza R, Molina S, Herrera J.Revisión de la literatura y definición de los criterios de admisión a la unidad de cuidado crítico obstétrico (intermedios - intensivos). Acta Colomb Cuid Intensivo. $2010 ; 10(1): 67-72$. 